\title{
INTERSECTING LEGENDRIANS AND BLOW-UPS
}

\author{
Andrew Hassell and András Vasy
}

\begin{abstract}
The purpose of this note is to describe the relationship between two classes of Legendre distributions. These two classes are distributions associated to an intersecting pair of Legendre submanifolds, introduced in [2] by analogy with intersecting Lagrangian distributions of Melrose and Uhlmann [8], and Legendre distributions associated to a fibred scattering structure introduced in [3]. We prove that, given appropriate symbolic orders, the first class is a proper subset of the second. We also give an example in two dimensions, which shows explicitly the relation between the two spaces in a simple setting.
\end{abstract}

\section{Introduction}

The purpose of the present note is to clarify the relationship between two classes of Legendre distributions. The first class, that of intersecting Legendrians associated to a pair of Legendre manifolds which intersect cleanly, was defined by one of us in [2] as an analog of the notion of intersecting Lagrangian distributions [8] of Melrose and Uhlmann. Just as the latter played an important role in the study of real principal type operators, the former proved useful in geometric scattering theory, both in describing the structure of the boundary value of the resolvent (of a scattering Laplacian) at the real axis [3] and in the study of three-body scattering [2].

The second class of Legendre distributions, that of Legendrians associated to a fibred-scattering structure, was defined in [3]. This extends the notion of Legendre distributions to manifolds with corners that are equipped with certain boundary fibrations, and it was used to analyze the structure of the resolvent of scattering differential operators near the the corners of the b-double space.

Our result is that, given appropriate geometry and symbolic orders, the class of intersecting Legendre distributions is a proper subset of Legendre distributions associated to a fibred scattering structure - see Theorem 3.2 in Section 3 for the precise statement. The proper inclusion corresponds to, roughly speaking, half of the possible terms in a Taylor series expansion of a general fibred-scattering Legendre distribution not being present in an intersecting Legendre distribution.

Lagrangian distributions on a manifold without boundary, $X_{0}$, are distributions on $X_{0}$ with very special singularities (in the sense of lack of smoothness) associated to Lagrangian submanifolds of the cotangent bundle of $X$. The simplest examples are conormal distributions to an embedded submanifold $Z \subset X_{0}$;

Received January 5, 2001. 
these are distributions whose regularity is maintained under repeated differentiation by vector fields tangent to $Z$; in particular they are smooth away from $Z$. Lagrangian distributions, and their generalizations, play a central role in modern PDE theory, see e.g. [5].

If $X_{0}$ is not compact, one can study decay/growth properties of distributions at 'infinity' in addition to studying their smoothness properties. Thus, the lack of rapid decay at infinity can be considered a 'singularity' and studied via microlocal analysis. Since one needs some structure at infinity, even to make sense of 'rapid decay', it is more natural to work on compact manifolds with boundary (or corners) which arise by the compactification of such $X_{0}$ and study singularities at the boundary. On manifolds with boundary, $X$, one can either introduce Legendre distributions from the symplectic (or really contact) point of view, as traditionally done for Lagrangian distributions, or instead simply write down such distributions and 'work backwards'. Since the former point of view, which is certainly 'neater', has been discussed in detail in [9], we follow the second approach. This should quickly make it clear that many familiar functions fall in the class of Legendre distributions.

Thus, let $X$ be a compact manifold with boundary, and $x$ a boundary defining function. Legendre distributions on $X$ are functions which are smooth in the interior of $X$ and have specific types of singularities at the boundary, which we shall now describe. Let $U$ be an open subset of $\partial X$, and consider the space $U \times \mathbb{R} \times \mathbb{R}^{n-1}$ with coordinates $y, \tau, \mu$. The form $\chi=d \tau+\mu \cdot d y$ is a contact form on $U \times \mathbb{R} \times \mathbb{R}^{n-1}$; that is, $\chi \wedge(d \chi)^{n-1}$ never vanishes. (Here we work in local coordinates, but this has an invariant geometric description in terms of the scattering cotangent bundle; see $[9,3]$ for a detailed description, and the next section for a brief summary.) Recall that a Legendre submanifold is a submanifold of maximal dimension (equal to $n-1$ ) on which the contact form vanishes.

Any Legendre submanifold $L$ of $U \times \mathbb{R} \times \mathbb{R}^{n-1}$ has a local parametrization, that is, a function $\phi(y, v)$, where $y \in U$ and $v \in \mathbb{R}^{p}$ such that locally

$L=\left\{(y, \tau, \mu) \mid \exists(y, v)\right.$ such that $\tau=-\phi(y, v), \mu=d_{y} \phi(y, v)$ and $\left.d_{v} \phi(y, v)=0\right\}$, and

for $1 \leq i \leq p, \quad d\left(\frac{\partial \phi}{\partial v_{i}}\right)$ are linearly independent.

Condition (1.1) ensures that the map

$$
\left\{(y, v) \mid d_{v} \phi(y, v)=0\right\} \rightarrow\left\{(y, \tau, \mu) \mid \tau=-\phi, \mu=d_{y} \phi\right\}
$$

is a diffeomorphism. The class $I_{\mathrm{sc}}^{m}(X, L)$ of Legendre distributions are defined as a finite sum of terms of the form

$$
x^{m+n / 4-p / 2} \int_{\mathbb{R}^{p}} e^{i \phi(y, v) / x} a(x, y, v) d v,
$$

where $\phi$ locally parametrizes $L$ and $a$ is smooth, with compact support in $v$. (We also allow the case that $\phi$ is a linear function of $v$ and $a$ is Schwartz in 
$v$; these are called 'extended Legendrian distributions' in [2].) We assume, of course, that $a$ is supported in the region where $\phi$ parametrizes $L$.

The microsupport of $u$ is the closed subset of $L$ corresponding, under (1.2), to the set

$$
\left\{(y, v) \mid d_{v} \phi=0 \text { and there is no neighbourhood of }(0, y, v)\right.
$$

in which $a$ is $\left.O\left(x^{\infty}\right)\right\}$.

By a partition of unity we can always write $u$ as a finite sum of terms each having microsupport as small as desired.

Let us give two examples. The simplest example is a function of the form

$$
u_{1}=x^{q} e^{i \phi(x, y) / x} a(x, y) \text {, with } \phi, a \text { smooth on } X .
$$

Here $(x, y)$ are coordinates on $X$, where $y=\left(y_{1}, \ldots, y_{n-1}\right)$ restrict to coordinates on $\partial X$. There is no loss of generality in assuming that $\phi$ depends only on $y$. The Legendre submanifold associated with $u_{1}$ in (1.3) is

$$
G_{1}=\left\{\left(y, \tau=-\phi(y), \mu=d_{y} \phi(y)\right)\right\} .
$$

The submanifold $G_{1}$ is essentially the 1 -jet of $\phi$. For each $m \in \mathbb{R}$, the class $I_{\mathrm{sc}}^{m}\left(X, G_{1}\right)$ is the class of functions $u_{1}$ of the form (1.3) with $q=m+n / 4$ and $a \in \mathcal{C}^{\infty}(X)$ arbitrary.

Another example is a function

$$
x^{q} V\left(x, \frac{y}{x}\right),
$$

where $V(x, w)$ is smooth in $x$ and Schwartz in $w \in \mathbb{R}^{n-1}$. More generally, if $y=\left(y^{\prime}, y^{\prime \prime}\right)$ is a splitting of the coordinates then a function of the form

$$
u_{2}=x^{q} V\left(x, \frac{y^{\prime}}{x}, y^{\prime \prime}\right)
$$

with $V\left(x, w^{\prime}, y^{\prime \prime}\right)$ smooth and Schwartz in $w^{\prime}$, is a Legendre distribution. The function $u_{2}$ can be written in terms of the Fourier transform in the $w$ variable as

$$
u_{2}=x^{q} \int e^{i y^{\prime} \cdot \eta^{\prime} / x} \hat{V}\left(x, \eta^{\prime}, y^{\prime \prime}\right) d \eta^{\prime} .
$$

The Legendre submanifold associated with $u_{2}$ is

$$
G_{2}=\left\{(y, \tau, \mu) \mid y^{\prime}=0, \tau=0, \mu^{\prime \prime}=0\right\},
$$

and it is easy to check that the function $y^{\prime} \cdot \eta$ parametrizes $G_{2}$, which is a sort of 'conormal bundle' to $C$; we call it the scattering conormal bundle and denote it ${ }^{\mathrm{sc}} N(C ; X)$. If $\operatorname{dim} y^{\prime}=k$ then the class $I_{\mathrm{sc}}^{m}\left(X, G_{2}\right)$ is the class of functions of the form (1.5) with $q=m+n / 4-k / 2$ and with $\hat{V}$ Schwartz in the second variable.

Such distributions turn up naturally in scattering theory. For example, let $X$ be the radial compactifiction of $\mathbb{R}^{n}$, and let $z$ be a linear coordinate on $\mathbb{R}^{n}$. 
Consider the function $e^{-i z \cdot k}, k \in \mathbb{R}^{n}$ which is a generalized eigenfunction of $\Delta$ with eigenvalue $|k|^{2}$. In inverted polar coordinates $(x, \hat{z}), x=|z|^{-1}, z=\hat{z} / x$, this function takes the form $e^{i \hat{z} \cdot k / x}$, which is a Legendre distribution of the first type. An example of a Legendre distribution of the second type is a function of some subset of the $z$ variables: let $z=\left(z^{\prime}, z^{\prime \prime}\right)$ and let $V$ be a Schwartz function of $z^{\prime}$. Then

$$
V\left(z^{\prime}\right)=(2 \pi)^{-k} \int e^{i \hat{z}^{\prime} \cdot \zeta^{\prime} / x} \hat{V}\left(\zeta^{\prime}\right) d \zeta^{\prime}
$$

is a Legendre distribution of the second type. Such functions appear naturally in the quantum $N$-body problem for example.

However, very frequently one comes across functions which are more complicated, and associated to more than one Legendre submanifold. For example, consider the kernel of the outgoing resolvent $R(\sigma+i 0)$ of the Laplacian on $\mathbb{R}^{3}$. This is

$$
G\left(\sigma, z, z^{\prime}\right)=\frac{1}{4 \pi} \frac{e^{i \lambda\left|z-z^{\prime}\right|}}{\left|z-z^{\prime}\right|}, \quad \lambda=\sqrt{\sigma}>0, \quad z, z^{\prime} \in \mathbb{R}^{3} .
$$

Let us multiply by a function $\chi\left(\left|z-z^{\prime}\right|\right)$ which is smooth, vanishes near $\left|z-z^{\prime}\right|=0$ and is $\equiv 1$ for $\left|z-z^{\prime}\right|>c$ to get rid of the interior singularity. (The difference is the kernel of a pseudodifferential operator, which is well understood.) Consider the resulting function $\chi G$, for fixed $\lambda>0$, as a function on the radial compactification $\tilde{X}$ of $\mathbb{R}^{6}$. Let $C \subset \partial \tilde{X}$ be the boundary of the diagonal $z=z^{\prime}$. Let us use coordinates $w=z-z^{\prime}$ and $w^{\prime}=\left(z+z^{\prime}\right) / 2$, and let $x=\left|w^{\prime}\right|^{-1}$. Then $x$ is a boundary defining function for $\tilde{X}$ near $C$, and local coordinates on $\partial \tilde{X}$ near $C$ are $y=\left(y^{\prime}, y^{\prime \prime}\right)$ where $y^{\prime}=x w \in \mathbb{R}^{3}$ and $y^{\prime \prime}=x w^{\prime} \in S^{2}$ (more precisely, $y^{\prime \prime}$ are local coordinates on $\left.S^{2}\right)$. This defines $C$ as $\left\{x=0, y^{\prime}=0\right\}$.

Away from $C$, we have $\left|z-z^{\prime}\right|=\phi(y) / x$ where $\phi(y)=\left|y^{\prime}\right|$. Thus away from $C, \phi$ is a smooth function and (1.7) is a Legendre distribution of the first type, associated to $L_{1}=\left\{\left(y,-\phi, d_{y} \phi\right)\right\}$. On the other hand, near the diagonal, $\chi G$ is a function of the form $V\left(y^{\prime} / x\right)$, which is a Legendre distribution of the second type, associated to $L_{2}={ }^{\mathrm{sc}} N(C ; X)=\left\{y^{\prime}=0, \tau=0, \mu^{\prime \prime}=0\right\}$. Clearly it is simultaneously associated to both of these Legendrian submanifolds.

Near $C, \phi$ is not a smooth function. Nevertheless, $L_{1}$ is a smooth Legendre submanifold, but with boundary. The boundary of $L_{1}$ is given by

$$
\partial L_{1}=\left\{y^{\prime}=0, y^{\prime \prime}, \tau=0,\left|\mu^{\prime}\right|=\lambda, \mu^{\prime \prime}=0\right\},
$$

which lies entirely over $C$ - in fact, it is contained in $L_{2}$. Also note that at $\left(0, y^{\prime \prime}, 0, \mu^{\prime}, 0\right) \in \partial L_{1}$, we have

$$
\mu^{\prime} \cdot d y^{\prime}=d\left(\mu^{\prime} \cdot y^{\prime}\right)=-d \tau=d \phi \neq 0 \text { in } L_{1},
$$

which implies that $L_{1}$ intersects $L_{2}$ cleanly at $L_{1} \cap L_{2}=\partial L_{1}$.

Now suppose that $L_{1}$ and $L_{2}$ are any two Legendre submanifolds, $L_{1}$ with boundary, such that $L_{1}$ intersects $L_{2}$ cleanly at $L_{1} \cap L_{2}=\partial L_{1}$. There are (at 
least) two ways of looking at distributions associated to $\left(L_{2}, L_{1}\right)$, and correspondingly, two classes of distributions that may be defined. The first way is to define intersecting Legendre distributions as was done in [2] (which is a routine generalization of the class of intersecting Lagrangian distributions to the Legendre setting). One defines a local parametrization of $\left(L_{2}, L_{1}\right)$ near a point $q \in L_{1} \cap L_{2}$ to be a function $\phi(y, v, s)$, where $v \in \mathbb{R}^{p}$ and $s \in[0, \infty)$, such that $\phi$ parametrizes $L_{1}$ in the sense analogous to (1.2) with both $v$ and $s$ taken as parameters, while $\phi(y, v, 0)$ parametrizes $L_{2}$. The nondegeneracy condition (1.1) is replaced by

$$
d\left(\frac{\partial \phi}{\partial s}\right), d\left(\frac{\partial \phi}{\partial v_{i}}\right) \text { and } d s \text { are linearly independent. }
$$

Then an expression of the form

$$
x^{m+n / 4-(p+1) / 2} \iint_{0}^{\infty} e^{i \phi(y, v, s) / x} a(x, y, v, s) d s d v,
$$

where $a$ is smooth, with compact support in $(s, v)$ and supported in the region where $\phi$ parametrizes $\left(L_{2}, L_{1}\right)$, is a Legendre distribution of order $m$ associated to $L_{1}$ away from $L_{2}$ (e.g. when $a$ is supported in $s \geq \epsilon>0$ ) and of order $m+1 / 2$ at $L_{2} \backslash L_{1}$ (to see this, multiply and divide by $d_{s} \phi / x$ and then integrate by parts in $s$ to get a boundary term at $s=0)$. The class $I_{\mathrm{sc}}^{m}\left(X,\left(L_{2}, L_{1}\right)\right)$ is defined to be those functions $u=u_{1}+u_{2}+u^{\prime}$, where $u_{1} \in I_{\mathrm{sc}}^{m}\left(X, L_{1}\right), u_{2} \in I_{\mathrm{sc}}^{m+1 / 2}\left(X, L_{2}\right)$ and $u^{\prime}$ given by a finite sum of terms of the form (1.9).

In the example (1.7) above, one can easily verify that

$$
w \cdot v-\left(|v|^{2}-\lambda^{2}\right) s, \quad v \in \mathbb{R}^{3}, \quad s \geq 0,
$$

parametrizes the pair $\left(L_{2}, L_{1}\right)$ associated with $\chi G$. It would be a good exercise for the reader to verify that $\chi G$ can indeed be written as an integral of the form (1.9), using the phase function (1.10).

The second way, when $L_{2}$ arises from an embedded submanifold $C \subset \partial X$ as above, involves blowing up the submanifold $C$ and defining fibred Legendrian distributions. Suppose $L_{1}$ is a Legendrian with boundary meeting $L_{2}$ cleanly at $\partial L_{1}=L_{1} \cap L_{2}$, and let $\tilde{L}_{1}$ be an extension of $L_{1}$ across the boundary to an open Legendre submanifold. Let $\left(y^{\prime}, y^{\prime \prime}\right)$ be coordinates on $\partial X$ which define $C$ as $\left\{x=0, y^{\prime}=0\right\}$, where $y^{\prime}=\left(y_{1}, \ldots, y_{k}^{\prime}\right)$. If we assume that $L_{1} \cap L_{2}$ has full rank projection to $C$, then this implies that near $q \in \partial L_{1}$, there must be one of the $y^{\prime}$ coordinates, say $y_{k}^{\prime}$, whose differential restricted to $L_{1}$ does not vanish at $q$ (this is shown in Section 5). Thus, on $L_{1}, y_{k}^{\prime}$ is a local boundary defining function near $q$. Since we have assumed that $\partial L_{1}$ has full rank projection to $C$, the span of the pull-back of the differentials $d y_{j}^{\prime}$ to $L_{1}$ at $q$ is exactly onedimensional. By a linear change of the $y^{\prime}$ coordinates we may assume that $d y_{1}^{\prime}, \ldots, d y_{k-1}^{\prime}$ pull back to 0 at $q$. Then standard contact arguments show that $y_{k}^{\prime}, y^{\prime \prime}$ and $v=\left(\mu_{1}^{\prime}, \ldots, \mu_{k-1}^{\prime}\right)$ give local coordinates on $\tilde{L}_{1}$ near $q$, and that $L_{2} \cap L_{1}$ is defined by $y_{k}^{\prime}=0$ in these coordinates. By switching the sign of $y_{k}^{\prime}$ if 

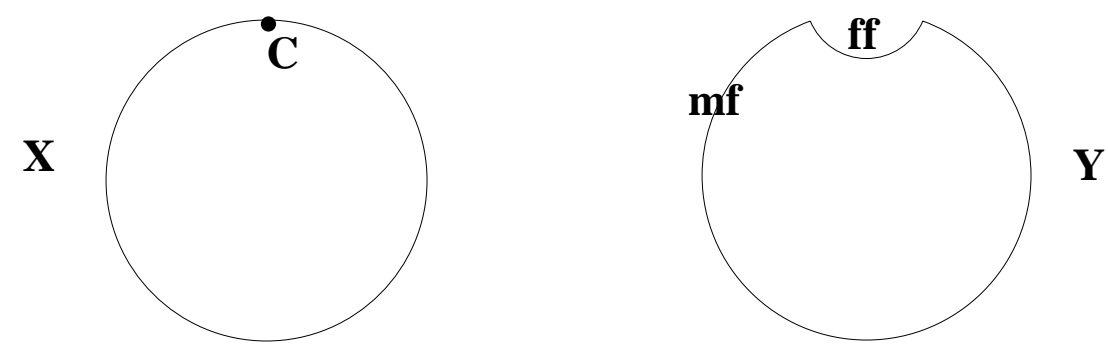

Figure 1. Blowing up $C \subset \partial X$ to produce $Y$.

necessary, we may also assume that microlocally $L_{1}$ lies in $y_{k}^{\prime} \geq 0$. Expressing $\tilde{y}=\left(y_{1}, \ldots, y_{k-1}\right)$ as $\tilde{y}=\tilde{Y}\left(y_{k}^{\prime}, y^{\prime \prime}, v\right), \tau$ as $\tau=T^{\prime}\left(y_{k}^{\prime}, y^{\prime \prime}, v\right)$ on $\tilde{L}_{1}$, it follows from general principles (see [5], volume 3 , Theorem 21.2.18, or [9], section 6) that a local nondegenerate parameterization of $\tilde{L}_{1}$ is given by

$$
\phi(y, v)=-T^{\prime}+v \cdot(\tilde{y}-\tilde{Y}), \quad v \in \mathbb{R}^{k-1} .
$$

Since $T^{\prime}=0$ and $\tilde{Y}=0$ at $\partial L_{1}$, and since $y_{k}^{\prime}$ is a boundary defining function for $L_{1}$, it follows that $\phi$ can be written in the form $y_{k}^{\prime} \tilde{\phi}\left(y^{\prime} / y_{k}^{\prime}, y_{k}^{\prime}, y^{\prime \prime}, v\right)$, with $\tilde{\phi}\left(z, y_{k}^{\prime}, y^{\prime \prime}, v\right)$ smooth near $z=y_{k}^{\prime}=0$.

We now interpret this in terms of blowing up the submanifold $C \subset X$. Let

$$
Y=[X ; C]
$$

be the manifold with codimension 2 corners obtained by real blowup of $C$. $Y$ has two boundary hypersurfaces: one which is the lift of $\partial X$, which we call the 'main face', and a new hypersurface arising from the blowup of $C$ which we call the 'front face'. These will be abbreviated $\mathrm{mf}$ and ff, respectively. Boundary defining functions are given by $\rho_{\mathrm{ff}}=\left(x^{2}+\left|y^{\prime}\right|^{2}\right)^{1 / 2}$ and $\rho_{\mathrm{mf}}=x / \rho_{\mathrm{ff}}$. Then $I_{\mathrm{sc}}^{m+1 / 2}\left(X, L_{2}\right)$ is identical with the set of functions of the form $x^{m+1 / 2-k / 2+n / 4} c$, where $c$ is smooth on $Y$ and vanishes to infinite order at mf, and $k$ is the codimension of $C$ in $\partial X$.

Let us define $L$ to be the closure of the lift of $\left(L_{1} \backslash \partial L_{1}\right) \subset X \times \mathbb{R} \times \mathbb{R}^{n-1}$ to $Y \times$ $\mathbb{R} \times \mathbb{R}^{n-1}$. It is a submanifold with boundary, contained in $\mathrm{mf} \times \mathbb{R} \times \mathbb{R}^{n-1}$, with $\partial L \subset(\mathrm{mf} \cap \mathrm{ff}) \times \mathbb{R} \times \mathbb{R}^{n-1}$. The submanifold $L$ is then a Legendre submanifold, and the function $\phi=y_{k}^{\prime} \tilde{\phi}$ a local parametrization of $L$, in the sense of [3]; note that $\tilde{\phi}$ is a smooth function near the corner of $Y$, since $y_{j}^{\prime} / y_{k}^{\prime}$ is smooth on $Y$ in the region of interest.

For the purposes of this paper, we define the class of fibred Legendrians of order $(m, r)$ associated to $L$, denoted $I_{\mathrm{s} \Phi}^{m, r}(Y, L)$, to be those functions $u=$ $u_{1}+u_{2}+u^{\prime}$, where $u_{1} \in I_{\mathrm{sc}}^{m}\left(X, L_{1}\right)$ and supported away from $C, u_{2} \in I_{\mathrm{sc}}^{r}\left(X, L_{2}\right)$ 
and $u^{\prime}$ given by a finite sum of terms of the form

$$
\begin{aligned}
y_{k}^{\prime r+\frac{n}{4}-\frac{k}{2}}\left(\frac{x}{y_{k}^{\prime}}\right)^{m+\frac{n}{4}-\frac{p}{2}} \int e^{i y_{k}^{\prime} \tilde{\phi}\left(\frac{y^{\prime}}{y_{k}^{\prime}}, y_{k}^{\prime}, y^{\prime \prime}, v\right) / x} a\left(y_{k}^{\prime}, \frac{y^{\prime}}{y_{k}^{\prime}}, \frac{x}{y_{k}^{\prime}}, y^{\prime \prime}, v\right) d v, \\
v \in \mathbb{R}^{p}
\end{aligned}
$$

in local coordinates. Here we assume that $y_{k}^{\prime} \tilde{\phi}$ locally parametrizes $L$ as above, $a$ is smooth on $Y$ and compactly supported, and that $a$ is supported near the corner of $Y$ (e.g. in $y_{k}^{\prime} \leq c, 0 \leq x / y_{k}^{\prime} \leq c$ ).

Notice that in the interior of $\mathrm{ff}$, say for $c<y_{k}^{\prime} / x<C, y_{k}^{\prime} / x$ is a smooth function on $Y$, so (1.12) gives something in $x^{r+n / 4-k / 2} \mathcal{C}^{\infty}(Y)$ in this region, which is consistent with the $u_{2}$ term being in $I_{\mathrm{sc}}^{r}\left(X, L_{2}\right)$.

One of the simplest examples of such a Legendre submanifold $L$ (though it does not arise from a Legendrian with boundary as in the setting above) is the zero section in $\operatorname{mf} \times \mathbb{R} \times \mathbb{R}^{n-1}$. This is parametrized by $\phi=0$, no $v$ variables are required, and $I_{\mathrm{s} \Phi}^{m, r}(Y, L)$ is identical with $\rho_{\mathrm{ff}}^{r+n / 4-k / 2} \rho_{\mathrm{mf}}^{m+n / 4-(k-1) / 2} \mathcal{C}^{\infty}(Y)$. In the case that $r=m+1 / 2$, this is the same as $x^{m+1 / 2+n / 4-k / 2} \mathcal{C}^{\infty}(Y)$.

The purpose of this paper is to clarify the relation between these two spaces $I_{\mathrm{sc}}^{m}\left(X,\left(L_{2}, L_{1}\right)\right)$ and $I_{\mathrm{s} \Phi}^{m, m+1 / 2}(Y, L)$ when $L_{2}={ }^{\mathrm{sc}} N(C ; X), Y=[X ; C]$, and $L$ is obtained from $L_{1}$ as described above.

\section{Invariant description}

To describe the situation more invariantly, let $X$ be a manifold with boundary, $n=\operatorname{dim} X$, and $x$ a boundary defining function for $X$. We denote the interior of $X$ by $X^{\circ}$. Then $X$ is naturally equipped with its scattering cotangent bundle, ${ }^{\mathrm{sc}} T^{*} X$. One way to describe ${ }^{\mathrm{sc}} T^{*} X$ is that its smooth sections are spanned, over $\mathcal{C}^{\infty}(X)$, by one-forms of the form $d(\phi / x), \phi \in \mathcal{C}^{\infty}(X)$. In particular, ${ }^{\mathrm{sc}} T_{X^{\circ}}^{*} X$ is naturally identified with $T^{*} X^{\circ}$. Let $p \in \partial X$ and let $(x, y)=\left(x, y_{1}, \ldots, y_{n-1}\right)$ be local coordinates near $p$. A local basis for ${ }^{\mathrm{sc}} T^{*} X$ near $p$ is given by $d x / x^{2}=$ $-d(1 / x)$ and $d y_{i} / x$. Thus, a point $q \in{ }^{\mathrm{sc}} T^{*} X$ may be written

$$
q=\tau \frac{d x}{x^{2}}+\sum_{i} \mu_{i} \frac{d y_{i}}{x}
$$

and this gives local coordinates $(x, y, \tau, \mu)$ on ${ }^{\mathrm{sc}} T^{*} X$, where $(\tau, \mu)$ are linear coordinates on each fibre. Moreover, ${ }^{\mathrm{sc}} T_{\partial X}^{*} X$ is naturally equipped with a contact structure via a contact form $\chi$ induced by the symplectic structure of $T^{*} X^{\circ}$, in a similar way to that in which a contact form is induced on the cosphere bundle $S^{*} X=\left(T^{*} X \backslash 0\right) / \mathbb{R}^{+}$. In the local coordinates given by (2.1), this form is equal to $\chi=d \tau+\mu \cdot d y$, as in the previous section.

Let $C$ be a closed embedded submanifold of $\partial X$. Suppose that $p \in C$, and that coordinates are chosen so that $C=\left\{x=0, y^{\prime}=0\right\}$ near $p$; here $y^{\prime}=$ $\left(y_{1}, \ldots, y_{k}^{\prime}\right)$. There is a well-defined 'scattering conormal bundle' over $C$, denoted ${ }^{\mathrm{sc}} N^{*}(C ; X)$, which is defined as the span of $d(\phi / x)$ (inside ${ }^{\mathrm{sc}} T_{C}^{*} X$ ) for all $\phi \in$ 
$\mathcal{C}^{\infty}(X)$ which vanish on $C$. In the local coordinates $\left(x, y^{\prime}, y^{\prime \prime}\right), \phi$ can be written as $\phi=\sum_{j} y_{j}^{\prime} a_{j}+x a_{0}$, so ${ }^{\mathrm{sc}} N^{*}(C ; X)$ is spanned by the $d y_{j}^{\prime} / x$, i.e. as a vector bundle over $C$ the dimension of each fibre is is the codimension of $C$ in $\partial X$. In local coordinates it is given by (1.6). It is easy to see that ${ }^{\mathrm{sc}} N^{*}(C ; X)$ is in fact a Legendre submanifold of ${ }^{\mathrm{sc}} T_{\partial X}^{*} X$. Note that $d x / x^{2}=-d(1 / x)$ does not lie in ${ }^{\text {sc }} N^{*}(C ; X)$. Morally speaking this is so because we are studying scattering one forms, and these are the differentials of functions that are singular at $\partial X$, so their behavior is quite different from that of the ordinary conormal bundle of $C$, spanned (inside $T_{C}^{*} X$ ) by the differentials of functions vanishing at $C$.

Let $Y=[X ; C]$ be the blow-up of $X$ at $C$, and let $\beta: Y \rightarrow X$ be the blow-down map. Let $\mathrm{mf}$ denote the lift of $\partial X$ to $Y$, and let $\mathrm{ff}$ denote the front face of the blow-up, i.e. the lift of $C$. Then $\mathrm{ff}$ has a natural fibration over $C$ given by the blow-down map: $\left.\beta\right|_{\mathrm{ff}}: \mathrm{ff} \rightarrow C$. As discussed in [3], this defines a structure algebra $\mathcal{V}_{\mathrm{s} \Phi}(Y)$ of vector fields, and more importantly for us, a corresponding replacement of the standard cotangent bundle, namely the scattering fibred cotangent bundle ${ }^{\mathrm{s} \Phi} T^{*} Y$. Sections of ${ }^{\mathrm{s} \Phi} T^{*} Y$ are spanned, over $\mathcal{C}^{\infty}(Y)$, by $d(\phi / x)$ where $\phi \in \mathcal{C}^{\infty}(Y)$ is constant on the fibers of the fibration. Such a setting is a natural generalization (to manifolds with corners) of the fibred cusp Lie algebra introduced by Mazzeo and Melrose on manifolds with a fibred boundary [7]. Then ${ }^{\mathrm{s} \Phi} T_{\mathrm{mf}}^{*} Y$ has a natural contact form which degenerates at the corner ${ }^{\mathrm{s} \Phi} T_{\mathrm{mf}}^{*} \cap \mathrm{ff} Y$. In this setting, ${ }^{\mathrm{s} \Phi} T^{*} Y$ is just the pull-back of ${ }^{\mathrm{sc}} T^{*} X$ by the blow-down map $\beta$, and we denote the induced map by $\tilde{\beta}:{ }^{\mathrm{s} \Phi} T^{*} Y \rightarrow{ }^{\mathrm{sc}} T^{*} X$. Thus, local coordinates on ${ }^{\mathrm{s} \Phi} T^{*} Y$ near the boundary consist of local coordinates on $Y$ together with the functions $\tau$ and $\mu$ lifted from ${ }^{\text {sc }} T^{*} X$. Moreover, the contact form on ${ }^{\mathrm{s} \Phi} T_{\mathrm{mf}}^{*} Y$ is just the pull-back of $d \tau+\mu \cdot d y$ by $\tilde{\beta}$. Given a Legendre submanifold $L \subset{ }^{\mathrm{s} \Phi} T_{\mathrm{mf}}^{*} Y$ which is transversal to the corner $\mathrm{mf} \cap \mathrm{ff}$ and satisfies a compatibility condition with the fibration, the class of Legendre distributions associated to $L$ was defined in [3]; in the case of interest here, the definition (1.12) above suffices.

\section{Main results}

To describe our main results, we let $L_{2}$ be the scattering conormal bundle ${ }^{\text {sc }} N^{*}(C ; X)$ as above, and suppose that $L_{1} \subset{ }^{\mathrm{sc}} T^{*} X$ is a Legendre submanifold with boundary which intersects $L_{2}$ cleanly in $\partial L_{1}=L_{2} \cap L_{1}$. In addition, we assume that $L_{2} \cap L_{1}$ has full rank projection to $C$. Thus, in local coordinates in ${ }^{\mathrm{sc}} T_{\partial X}^{*} X, L_{2}=\left\{y^{\prime}=0, \tau=0, \mu^{\prime \prime}=0\right\}$. We shall prove in Lemma 5.1 that these assumptions imply that $L_{1}$ also intersects ${ }^{\mathrm{sc}} T_{C}^{*} X$ cleanly, with intersection $L_{2} \cap L_{1}$, and in particular that at any $q \in L_{1}$, the pull-back of $d y_{j}^{\prime}$ to $L_{1}$ does not vanish for some $j$. Without loss of generality we may assume that the restriction of $d y_{k}^{\prime}$ to $L_{1}$ does not vanish at $q$; thus, near $q$, we may assume that $y_{k}^{\prime} \geq 0$ is a boundary defining function for $L_{1}$. This implies that under the blow-down map $\beta^{*}:{ }^{\mathrm{s} \Phi} T_{\mathrm{mf}}^{*} Y \rightarrow{ }^{\mathrm{sc}} T_{\partial X}^{*} X, L_{1}$ lifts to a Legendre submanifold $L$ which is transversal to ${ }^{\mathrm{s} \Phi} T_{\mathrm{mf} \cap \mathrm{ff}}^{*} Y$ and has full rank projection to $C$. 
Let $\tilde{L}_{1}$ be an extension of $L_{1}$ across its boundary to an (open) Legendre submanifold. Our first result is a characterization of $I_{\mathrm{sc}}^{m}\left(X,\left(L_{2}, L_{1}\right)\right)$ in terms of $I^{m}\left(X, \tilde{L}_{1}\right)$.

Theorem 3.1. Suppose $L_{1}$ and $L_{2}$ are as above, and let $\alpha \in \mathcal{C}^{\infty}(\mathbb{R})$ be such that $\alpha(t)$ is identically 1 for $t>1$, and identically 0 for $t<0$. Let $U$ be $a$ neighborhood of $q \in L_{2} \cap L_{1}$ in ${ }^{s c} T_{\partial X}^{*} X$ such that $y_{k}^{\prime}$ is a defining function for $\partial L_{1}$ in $L_{1} \cap U$. Intersecting Legendre distributions $u \in I_{s c}^{m}\left(X,\left(L_{2}, L_{1}\right)\right)$ microsupported in $U$ may be represented as

$$
\alpha\left(\frac{y_{k}^{\prime}}{x}\right) u_{1}+u_{0}
$$

where $u_{1} \in I_{s c}^{m}\left(X, \tilde{L}_{1}\right)$ and $u_{0} \in I_{s c}^{m+1 / 2}\left(X, L_{2}\right)$. Conversely, any such function is in $I_{s c}^{m}\left(X,\left(L_{2}, L_{1}\right)\right)$.

Note that $\alpha$ can be replaced by any other $\mathcal{C}^{\infty}$ function on $\mathbb{R}$ which is 1 on $[T,+\infty), 0$ on $(-\infty,-T], T>0$ sufficiently large, since the difference between the corresponding first terms in (3.1) can be absorbed in $u_{0}$.

Given this theorem we can rather easily understand the relation between the spaces $I_{\mathrm{sc}}^{m}\left(X,\left(L_{2}, L_{1}\right)\right)$ and $I_{\mathrm{s} \Phi}^{m, m+1 / 2}(Y, L)$, where $Y=[X ; C]$ and $L$ is obtained from $L_{1}$ as described in Section 1 .

Theorem 3.2. The space $I_{s c}^{m}\left(X,\left(L_{2}, L_{1}\right)\right)$ is a proper subset of $I_{\mathrm{s} \Phi}^{m, m+1 / 2}(Y, L)$. In particular, $I_{\mathrm{s} \Phi}^{m, m+1 / 2}(Y, L)$ is invariant under multiplication by $\mathcal{C}^{\infty}(Y)$ functions, while $I_{s c}^{m}\left(X,\left(L_{2}, L_{1}\right)\right)$ is not.

Remark. One can show that the algebraic $\mathcal{C}^{\infty}(Y)$ module generated by the space $I_{\mathrm{sc}}^{m}\left(X,\left(L_{2}, L_{1}\right)\right)$ is dense in $I_{\mathrm{s} \Phi}^{m, m+1 / 2}(Y, L)$. Hence, one could say that the space $I_{\mathrm{s} \Phi}^{m, m+1 / 2}(Y, L)$ is generated over $\mathcal{C}^{\infty}(Y)$ by $I_{\mathrm{sc}}^{m}\left(X,\left(L_{2}, L_{1}\right)\right)$.

Remark. Guillemin and Uhlmann [1] and Joshi [6] have defined paired Lagrangian distributions of independent orders $(m, r)$ associated to a pair $\left(L_{1}, L_{2}\right)$ with the geometry as described above. Using the Fourier transform and a local identification of neighbourhoods of $p \in \partial X$ with cones in $\mathbb{R}^{n}$, one can define spaces of paired Legendre distributions $I_{\mathrm{sc}}^{r, m}\left(X,\left(L_{2}, L_{1}\right)\right)$. In our setting these are defined microlocally near $\partial L_{1}$ by replacing $(1.9)$ by

$$
x^{m+n / 4-(p+1) / 2} \iint_{-\infty}^{\infty} s_{+}^{r-(m+1 / 2)} e^{i \phi(y, v, s) / x} a(x, y, v, s) d s d v,
$$

i.e. the distribution $s_{+}^{r-(m+1 / 2)}$ replaces the Heaviside step function $H(s)=$ $s_{+}^{0}$. Then when $r-\left(m+\frac{1}{2}\right)$ is a nonnegative integer, Theorem 3.2 generalizes directly, that is, distributions of the form (3.2) form a proper subset of the class $I_{\mathrm{s} \Phi}^{m, r}(Y, L)$. However, when $r-\left(m+\frac{1}{2}\right)$ is not an integer, then the two spaces coincide:

$$
I_{\mathrm{sc}}^{r, m}\left(X,\left(L_{2}, L_{1}\right)\right)=I_{\mathrm{s} \Phi}^{m, r}(Y, L), \quad r-\left(m+\frac{1}{2}\right) \notin \mathbb{Z}
$$


We prove (3.3) in a model case only, in the next section.

Remark. It is very natural to assume that $L_{1} \cap L_{2}$ is codimension one in $L_{2}$ and in $L_{1}$ since this appears in real principal type propagation. However, one can also consider the case of higher codimension intersections. In the case of Lagrangian manifolds, such intersections were studied by Guillemin and Uhlmann [1]. They essentially define the associated class of distributions by blowing up the intersection $L_{2} \cap L_{1}$. Note that if the intersection has codimension one, the blow-up divides $L_{1}$ into two manifolds with boundary, each with boundary $L_{2} \cap L_{1}$, hence resulting exactly in the setting discussed above. In our case thus the most natural definition of such intersecting Legendre distributions associated to an intersecting pair $\left(L_{2}, L_{1}\right)$ with $L_{2} \cap L_{1}$ having codimension greater than one is via the fibred scattering structure rather than directly by oscillatory integrals. In particular, if $L_{2}={ }^{\mathrm{sc}} N^{*}(C ; X), L_{1}$ is the zero section of ${ }^{\mathrm{sc}} T_{\partial X}^{*} X$, the codimension of the intersection $L_{2} \cap L_{1}$ (in $L_{2}$ and in $L_{1}$ ) is given by the dimension of the fibers of ${ }^{\mathrm{sc}} N^{*}(C ; X) \rightarrow C$, namely by the codimension of $C$ in $\partial X$. The natural definition of the class $I_{\mathrm{sc}}^{m}\left(X,\left(L_{2}, L_{1}\right)\right)$ of distributions associated to the intersecting Legendre pair $\left(L_{2}, L_{1}\right)$ is functions of the form $x^{m+\operatorname{dim} X / 4} \alpha\left(\left|y^{\prime}\right| / x\right) f+g$, $f \in \mathcal{C}^{\infty}(X), g \in \mathcal{C}^{\infty}([X ; C])$ with infinite order vanishing on $\mathrm{mf}$.

\section{An example}

Although the general case is hardly more complicated, for the sake of clarity we first consider the case when $\operatorname{dim} X=2$, and $C \subset \partial X$ is a point $x=0, y=0$, and $L_{1}$ is the zero section of ${ }^{\mathrm{sc}} T^{*} X$ in $y \geq 0$. The lift $L$ of $L_{1}$ to ${ }^{\mathrm{s} \Phi} T_{\mathrm{mf}}^{*} Y$ is the zero section then. Thus, fibred Legendrians in $I_{\mathrm{s} \Phi}^{m, m+1 / 2}(Y, L)$ are $\mathcal{C}^{\infty}$ functions on $Y=[X ; C]$, multiplied by powers of the boundary defining function, i.e. functions of the form $x^{m+1 / 2} c, c \in \mathcal{C}^{\infty}(Y)$. Let us show that elements $u$ of $I_{\mathrm{sc}}^{m}\left(X,\left(L_{2}, L_{1}\right)\right)$ satisfy

(4.1) $u \in I_{\mathrm{sc}}^{m}\left(X,\left(L_{2}, L_{1}\right)\right) \Leftrightarrow u=x^{m+1 / 2} c$, where $c=\alpha\left(\frac{y}{x}\right) f(x, y)+g\left(x, \frac{y}{x}\right)$.

Here $f$ is smooth, $\alpha$ is as in Theorem 3.1, and $g(x, Z)$ is a smooth function of $x$ taking values in Schwartz functions of $Z$. Away from the corner of $Y, c$ in (4.1) is an arbitrary element of $\mathcal{C}^{\infty}(Y)$. However, at the corner, its Taylor series is restricted so that it only has terms of the form $y^{j}(x / y)^{k}$ with $j \geq k$. (To see this, note that $g$ is zero to all orders in Taylor series at the corner, while $\alpha$ is equal to the constant function 1 to all orders at the corner. As for $f$, it has an arbitrary Taylor series in powers of $x$ and $y$, which amounts to a Taylor series in $x / y$ and $y$ subject to the constraint above.) Establishing (4.1) thus proves Theorems 3.1 and 3.2 in this case.

A parametrization of $\left(L_{2}, L_{1}\right)$ in this case is given by $\zeta(y-\bar{y})$, where $\zeta$ plays the role of $v$, and $\bar{y}$ that of $s$, in (1.9). Therefore, an intersecting Legendre distribution, $u \in I_{\mathrm{sc}}^{m}\left(X,\left(L_{2}, L_{1}\right)\right)$, associated to these Legendrians is one which 
can be written as $u=u_{0}+u_{1}+u^{\prime}$, with $u_{0} \in I_{\mathrm{sc}}^{m+1 / 2}\left(X, L_{2}\right), u_{1} \in I_{\mathrm{sc}}^{m}\left(X, L_{1}\right)$, microsupported away from $\partial L_{1}$ and $u^{\prime}$ of the form

$$
x^{m-1 / 2} \int_{0}^{\infty} \int e^{i \zeta \cdot(y-\bar{y}) / x} a(x, y, \zeta, \bar{y}) d \zeta d \bar{y}
$$

where $a$ has compact support in $x, y, \bar{y}$, and is Schwartz in $\zeta$. Directly from the definition, $u_{1}$ is of the form $x^{m+1 / 2} f(x, y)$, where $f$ is rapidly decreasing at the boundary wherever $y<0$. On the other hand, it is easy to see that $u_{0}$ is in $x^{m+1 / 2} \mathcal{C}^{\infty}(Y)$, vanishing to infinite order at the main face. Indeed, by definition, $u_{0}$ can be written as

$$
x^{m+1 / 2} \int e^{i \zeta y / x} a(x, y, \zeta) d \zeta,
$$

with $a$ compactly supported in $x, y$, rapidly decreasing in $\zeta$. But this is just Fourier transform in $\zeta$, hence the result is of the form $x^{m+1 / 2} b(x, y, y / x)$, with compact support in $x, y$ and rapid decay in $y / x$, which implies infinite order vanishing at the main face. Hence the real question is whether functions as in (4.2) are of the form (4.1), and conversely, whether such functions supported near the corner can be written as in (4.2) modulo $I_{\mathrm{sc}}^{m+1 / 2}\left(X, L_{2}\right)+I_{\mathrm{sc}}^{m}\left(X, L_{1}\right)$.

Start with the former, i.e. consider an oscillatory integral of the form (4.2). Since by assumption $a$ is compactly supported in $y$ and $\bar{y}$, we may suppose that $a$ is supported in $y, \bar{y} \leq c$. Let $\psi(\bar{y})$ be a compactly supported function which is equal to 1 for $0 \leq y \leq 2 c$, so that $a=a \psi(\bar{y})$.

First we claim that we may, modulo an $O\left(x^{\infty}\right)$ error, assume that $a$ is of the form

$$
a(x, y, \zeta, \bar{y})=a_{1}(x, y, \zeta) \psi(\bar{y}) .
$$

The reason is that we can expand $a$ as a Taylor series around $y=\bar{y}$ :

$$
a(x, y, \bar{y}, \zeta)=a(x, y, y, \zeta)+(y-\bar{y}) \tilde{a}(x, y, \bar{y}, \zeta) .
$$

Since $(y-\bar{y})=\partial_{\zeta} \phi$, we gain a power of $x$ by integrating by parts in $\zeta$ so as to get rid of this factor. Doing this repeatedly and asymptotically summing the series so obtained we obtain, modulo an $O\left(x^{\infty}\right)$ error, a function $a_{1}$ independent of $\bar{y}$.

Thus performing the $\bar{y}$ integral followed by the $\zeta$ integral, we get

$$
\begin{gathered}
x^{m-1 / 2} \int_{-\infty}^{\infty} \int_{-\infty}^{\infty} e^{i \zeta \cdot(y-\bar{y}) / x} a_{1}(x, y, \zeta) H(\bar{y}) \psi(\bar{y}) d \zeta d \bar{y} \\
=x^{m-1 / 2} \int_{\infty}^{\infty} e^{i \zeta \cdot y / x} a_{1}(x, y, \zeta) \widehat{H \psi}(\zeta / x) d \zeta \\
=x^{m-1 / 2} \int_{-\infty}^{\infty} H(y-s) \psi(y-s) \check{a}_{1}\left(x, y, \frac{s}{x}\right) d s
\end{gathered}
$$

Here $H$ is the Heaviside function and we have written $\check{a}_{1}$ for the inverse Fourier transform in the third variable; $\check{a}_{1}$ is a Schwartz function in its third variable 
(since it is the Fourier transform of a Schwartz function in that variable). We may write

$$
\check{a}_{1}(x, y, Z)=d(x, y) \alpha^{\prime}(Z)+b(x, y, Z),
$$

where $\alpha$ is as in Theorem 3.1, $d$ is smooth, $b$ is Schwartz in $Z, d$ and $b$ are both supported in $y \leq c$, and $\int b d Z=0$. (To do this, we take $d(x, y)=$ $\int \check{a}_{1}\left(x, y, Z^{\prime}\right) d Z^{\prime}$.) Thus, the integral

$$
g(x, y, Z)=\int_{-\infty}^{Z} b\left(x, y, Z^{\prime}\right) d Z^{\prime}
$$

is a Schwartz function of $Z$. Substituting this into (4.3), and writing $Z=y / x$, we get

$$
(4.2)=x^{m+1 / 2} \int_{-\infty}^{Z} \psi\left(y-x Z^{\prime}\right)\left(d(x, y) \alpha^{\prime}\left(Z^{\prime}\right)+b\left(x, y, Z^{\prime}\right)\right) d Z^{\prime} .
$$

Since $d$ and $b$ are both supported in $y \leq c, \psi=1$ on $[0,2 c]$, and $\alpha^{\prime}$ and $b$ are Schwartz in $Z^{\prime}$, we may, modulo $O\left(x^{\infty}\right)$, replace $\psi$ by 1 in this equation. This yields

$$
x^{m+1 / 2}\left(d(x, y) \alpha\left(\frac{y}{x}\right)+g\left(x, y, \frac{y}{x}\right)\right) .
$$

Expanding $g$ as a Taylor series in the second variable we see that we have expressed (4.2) in the form (4.1).

Conversely, let $u$ be of the form (4.1). The $g$ term is Legendrian with respect to $L_{2}$, so we only need deal with the first term. In fact, it is clear that the class $I_{\mathrm{sc}}^{m}\left(X,\left(L_{2}, L_{1}\right)\right)$ is invariant under multiplication by smooth functions on $X$, so we need only treat the $\alpha$ function. We may localize near $x=0, y=0$ by multiplying $\alpha(y / x)$ by $\chi(x, y)$, where $\chi \in \mathcal{C}_{c}^{\infty}\left(\mathbb{R}^{2}\right), \chi(x, y) \equiv 1$ in a neighbourhood of $(0,0)$ and is supported in $|y| \leq c,|x| \leq c$ say. Then for $\psi \in \mathcal{C}_{c}^{\infty}(\mathbb{R})$, identically 1 on $[-2 c, 2 c]$, the identity $\chi(x, y) \alpha^{\prime}((y-\bar{y}) / x)=\psi(\bar{y}) \chi(x, y) \alpha^{\prime}((y-\bar{y}) / x)$ holds. Indeed, on $\operatorname{supp} \alpha^{\prime},|y-\bar{y}| \leq x \leq c$, hence on $\operatorname{supp}\left(\chi \alpha^{\prime}\right),|\bar{y}| \leq 2 c$. Thus,

$$
\begin{gathered}
\chi(x, y) \alpha\left(\frac{y}{x}\right)=x^{-1} \chi(x, y) \int_{0}^{\infty} \alpha^{\prime}\left(\frac{y-\bar{y}}{x}\right) d \bar{y} \\
=x^{-1} \chi(x, y) \int_{0}^{\infty} \psi(\bar{y}) \alpha^{\prime}\left(\frac{y-\bar{y}}{x}\right) d \bar{y} .
\end{gathered}
$$

We can write this as

$$
x^{-1} \chi(x, y) \int_{-\infty}^{\infty} d \zeta \int_{0}^{\infty} e^{i(y-\bar{y}) \zeta / x} \psi(\bar{y}) \widehat{\alpha^{\prime}}(\zeta) d \bar{y}
$$

which is of the right form since $\alpha^{\prime}$, and therefore also its Fourier transform, is Schwartz.

We now discuss the spaces $I_{\mathrm{sc}}^{r, m}\left(X,\left(L_{2}, L_{1}\right)\right)$ in our model situation. A function $u \in I_{\mathrm{sc}}^{r, m}\left(X,\left(L_{2}, L_{1}\right)\right)$ can be written $u=u_{0}+u_{1}+u^{\prime}$, with $u_{0} \in I_{\mathrm{sc}}^{r}\left(X, L_{2}\right)$, 
$u_{1} \in I_{\mathrm{sc}}^{m}\left(X, L_{1}\right)$, microsupported away from $\partial L_{1}$ and $u^{\prime}$ of the form

$$
x^{m-1 / 2} \int_{0}^{\infty} \int \bar{y}^{r-m-1 / 2} e^{i \zeta \cdot(y-\bar{y}) / x} a(x, y, \zeta, \bar{y}) d \zeta d \bar{y}
$$

where $a$ has compact support in $x, y, \bar{y}$, and is Schwartz in $\zeta$. There is no difficulty in dealing with the $u_{0}$ and $u_{1}$ pieces, so we focus on the integral (4.5). Let $q=r-\left(m+\frac{1}{2}\right)$.

Let us first show that $I_{\mathrm{sc}}^{r, m}\left(X,\left(L_{2}, L_{1}\right)\right) \subset I_{\mathrm{s} \Phi}^{m, r}(Y, L)$. We start with (4.5) and apply the argument between (4.2) and (4.3), obtaining

$$
x^{m-1 / 2} \int_{-\infty}^{\infty}(y-s)_{+}^{q} \psi(y-s) \check{a}_{1}\left(x, y, \frac{s}{x}\right) d s
$$

instead of the last line of (4.3). This can be written

$$
x^{m+q+1 / 2} \int_{-\infty}^{\infty}\left(\frac{y}{x}-Z^{\prime}\right)_{+}^{q} \psi\left(y-x Z^{\prime}\right) \check{a}_{1}\left(x, y, Z^{\prime}\right) d Z^{\prime} .
$$

It is straightforward to check that this is in $x^{m+1 / 2} \rho_{\mathrm{ff}}^{q} \mathcal{C}^{\infty}(Y)$ away from the corner of $Y$. To see how this behaves near the corner, where $x / y=0$, we write

$$
\left(\frac{y}{x}-Z^{\prime}\right)^{q}=\left(\frac{y}{x}\right)^{q}\left(1+Z^{\prime} \frac{x}{y}\right)^{q}
$$

and expand the second term in a Taylor series at $x / y=0$. This can be done to any order, in spite of the factor $Z^{\prime}$, since $\check{a}_{1}\left(x, y, Z^{\prime}\right)$ is Schwartz in $Z^{\prime}$. Also, as before, we may replace $\psi$ by 1 at the cost of an $O\left(x^{\infty}\right)$ error. Integration in $Z^{\prime}$ then yields a function of the form

$$
x^{m+1 / 2} y^{q} c\left(x, y, \frac{x}{y}\right)
$$

where $c$ is smooth near the corner. Hence this term is in $x^{m+1 / 2} \rho_{\mathrm{ff}}^{q} \mathcal{C}^{\infty}(Y) \equiv$ $I_{\mathrm{s} \Phi}^{m, r}(Y, L)$.

Here we can see that something different happens when $q$ is a nonnegative integer. Namely, in the integral case the Taylor series expansion has only a finite number of terms, so $c$ has only a finite Taylor series in $x / y$ then, reflecting the restriction on the Taylor series at the corner mentioned earlier.

Finally we show, when $q$ is not an integer, that every element of $I_{\mathrm{s} \Phi}^{m, r}(Y, L)$ is in $I_{\mathrm{sc}}^{r, m}\left(X,\left(L_{2}, L_{1}\right)\right)$. To do this, it is enough to find an expression (4.5) such that the corresponding $c(x, y, x / y)$ as in (4.6) above has an arbitrary Taylor series in $x / y$ at $x=y=0$. The coefficient of $(x / y)^{j}$ in this series is given by

$$
\left(\begin{array}{l}
q \\
j
\end{array}\right) \int_{-\infty}^{\infty} Z^{\prime j} \check{a}_{1}\left(0,0, Z^{\prime}\right) d Z^{\prime}=\left(\begin{array}{l}
q \\
j
\end{array}\right) D_{\zeta}^{j} a_{1}(0,0, \zeta)\lceil\zeta=0 .
$$

If $q$ is nonintegral, then the combinatorial factor is nonzero, and it is clear that $a_{1}$ may be chosen so that these coefficients are arbitrary. 


\section{The general case}

Let $X, n, C, Y, L_{2}, L_{1}, \tilde{L}_{1}$, and $L$ be as in section 3 , and let $q \in \partial L_{1}$. Let $\tilde{L}_{1}$ be a Legendrian extension of $L_{1}$ to a submanifold without boundary across $L_{2}$. In local coordinates, $C=\left\{x=0, y^{\prime}=0\right\}$ and $L_{2}=\left\{x=0, y^{\prime}=0, \mu^{\prime \prime}=0\right\}$. First we prove a statement asserted just before the main results from section 3 .

Lemma 5.1. There is a $y^{\prime}$ coordinate, which may be taken to be $y_{k}^{\prime}$ without loss of generality, whose differential restricted to $L_{1}$ does not vanish at $q$.

Proof. Let $q \in L_{2} \cap L_{1}$, and let $W=T_{q}\left(L_{2} \cap L_{1}\right) \subset Z=T_{q}^{\mathrm{sc}} T_{\partial X}^{*} X$. Moreover, let $V_{j}=T_{q} L_{j} \subset Z$. The fact that a subspace $V$ of $Z$ is Legendre means that both $\chi$ and $d \chi$ vanish on it identically, i.e. $\chi(v)=0, d \chi\left(v, v^{\prime}\right)=0$ for all $v, v^{\prime} \in V$, and $\operatorname{dim} V=n-1$. Note that $d \chi$ is non-degenerate on $\operatorname{Ker} \chi \subset Z$, i.e. it is a symplectic form on this vector space.

Now, both $\chi$ and $d \chi$ vanish on $W$ since $W \subset V_{2}$. Let $W^{\prime}$ denote the subspace of Ker $\chi$ which annihilates $W$, i.e. $W^{\prime}=\left\{w^{\prime} \in Z: \chi\left(w^{\prime}\right)=0, d \chi\left(w^{\prime}, w\right)=\right.$ 0 for all $w \in W\}$. Then any Legendre subspace $V \supset W$ of $Z$ satisfies $V \subset W^{\prime}$ since $V \subset \operatorname{Ker} \chi$ and $d \chi(v, w)=0$ for all $w \in W \subset V$. Note that $W$ has codimension 2 in $W^{\prime}$ (since $W$ has codimension 1 in the Legendre subspace $V_{2}$ ). Thus, $W^{\prime} / W$ is a 2 -dimensional vector space, and $d \chi$ descends to a symplectic form on it. The image $V^{\prime}$ of a Legendre subspace $V \supset W$ in $W^{\prime} / W$ is Lagrangian with respect to this form. There is a one-dimensional family of such Lagrangian subspaces; the image of $V_{2}$ is one of them. Indeed, given any non-zero element $u$ of $W^{\prime} / W$, there is a unique Lagrangian subspace of $W^{\prime} / W$ which includes $u$, namely the span of $u$. This then determines a unique Legendre subspace $V$ of $W^{\prime}$ with $W \subset V$.

We claim that $W^{\prime}$ is not a subspace of $T_{q}{ }^{\mathrm{sc}} T_{C}^{*} X$. Indeed, suppose otherwise, i.e. that $W^{\prime} \subset T_{q}{ }^{\mathrm{sc}} T_{C}^{*} X$. The hypothesis on the full rank projection of $L_{2} \cap L_{1}$ means that $d y_{j}^{\prime \prime}$ are independent on $W$. The corresponding Hamilton vectors $\partial_{\mu_{j}^{\prime \prime}}$ under $d \chi$ in Ker $\chi$ are tangent to ${ }^{\mathrm{sc}} T_{C}^{*} X$, hence in $T_{q}{ }^{\mathrm{sc}} T_{C}^{*} X \cap \operatorname{Ker} \chi$. Thus, they span a $(\operatorname{dim} C)$-dimensional subspace $T$ of this space. If $f$ is a nonzero linear combination of the functions $y_{j}^{\prime \prime}$, then $d f$ does not vanish on $W$, which implies that $d \chi\left(H_{f}, \cdot\right)$ does not vanish on $W$. Hence $H_{f} \notin W^{\prime}$, which means that $T$ and $W^{\prime}$ have trivial intersection. But this is a contradiction: by dimension counting, the codimension of $W^{\prime}$ in $T_{q}{ }^{\mathrm{sc}} T_{C}^{*} X \cap \operatorname{Ker} \chi$ is $\operatorname{dim} C-1$. Hence $W^{\prime}$ is not a subspace of $T_{q}{ }^{\mathrm{sc}} T_{C}^{*} X$.

Thus $d y_{j}^{\prime}$ cannot all vanish identically on $W^{\prime}$. Since they all vanish identically on $V_{2}$, this is the only Legendre subspace with this property. By the clean intersection assumption, $V_{2} \cap V_{1}=W$, i.e. $V_{2}$ and $V_{1}$ are not the same. Hence the $d y_{j}^{\prime}$ do not all vanish on $V_{1}$, i.e. the pull-back of $d y_{j}^{\prime}$ to $L_{1}$ at $q$ is non-zero for some $j$. By relabelling the coordinates, we may assume that $d y_{k}^{\prime}$ is non-zero.

As discussed in Section 1, $\tilde{L}_{1}$ has a local parametrization of the form (1.11). This is also a local parametrization of $L$. A local nondegenerate parametrization 
of $\left(L_{2}, L_{1}\right)$ is given by

$$
\psi(y, v, \zeta, \bar{y})=-T^{\prime}+v \cdot(\tilde{y}-\tilde{Y})+\zeta\left(y_{k}^{\prime}-\bar{y}\right), \quad \bar{y} \geq 0
$$

where $\zeta$ plays the role of $v$, and $\bar{y}$ that of $s$, in (1.9).

We are now ready to prove Theorems 3.1 and 3.2 .

Proof of Theorem 3.1. Write $u \in I_{\mathrm{sc}}^{m}\left(X,\left(L_{2}, L_{1}\right)\right)$ in terms of the phase function $\psi$ from (5.1). We may then run the argument in the example of the previous section in the variables $\left(x, y_{k}^{\prime}, \bar{y}, \zeta\right)$ to show that $u$ can be written in the form (3.1). To prove the converse, write $u_{1}$ with respect to the phase (1.11) and express $\alpha$ as in (4.3) to obtain an expression involving the phase function $\psi$ from (5.1), which is manifestly an element of $I_{\mathrm{sc}}^{m}\left(X,\left(L_{2}, L_{1}\right)\right)$.

Proof of Theorem 3.2. First we show that $I_{\mathrm{sc}}^{m}\left(X,\left(L_{2}, L_{1}\right)\right)$ is contained in $I_{\mathrm{s} \Phi}^{m, m+1 / 2}(Y, L)$. Using Theorem 3.1 we may write $u \in I_{\mathrm{sc}}^{m}\left(X,\left(L_{2}, L_{1}\right)\right)$ in the form (3.1). In terms of this representation, $u_{0}$ is $x^{m+n / 4-(k-1) / 2}$ times an element of $\mathcal{C}^{\infty}(Y)$ which vanishes to all orders at the main face, so this is certainly an element of $I_{\mathrm{s} \Phi}^{m, m+1 / 2}(Y, L)$ (in fact, $I_{\mathrm{s} \Phi}^{r, m+1 / 2}(Y, L)$ for any $r$ ). On the other hand, $u_{1}$ is in $I_{\mathrm{sc}}^{m}\left(X, \tilde{L}_{1}\right)$ so can be written with respect to the phase function $\phi$ from (1.11). But this is also a phase function for $L$, and multiplication by a suitable $\alpha$ means it is now supported in $y_{k}^{\prime} / x>C$, so $\alpha \cdot u_{1}$ is also in $I_{\mathrm{s} \Phi}^{m, m+1 / 2}(Y, L)$.

To show that the inclusion is proper, we write $u$ in terms of a reduced symbol and show that its Taylor series is restricted at $x / y_{k}^{\prime}=y_{k}^{\prime}=0$, that is, at the intersection of the front face and the main face on $Y$. Using standard arguments, we can write $u$ in the form (1.12) using the parametrization from (5.1), where $a$ only depends on $y_{k}^{\prime}, x / y_{k}^{\prime}, y^{\prime \prime}$ and $v=\left(\mu_{1}^{\prime}, \ldots, \mu_{k-1}^{\prime}\right)$. (To reduce $a$ so as to depend on only these variables we repeatedly integrate by parts as done in the previous section for example.) Note that when $r=m+1 / 2$ and $p=k-1$, then the power of $y_{k}^{\prime}$ outside the integral in (1.12) vanishes. By the symbol calculus of [4], the reduced symbol is determined to all orders in Taylor series at $\mathrm{mf} \cap \mathrm{ff}$ by $u$. Using the description given by Theorem 3.1 , we see that the $u_{0}$ term has trivial Taylor series at $x / y_{k}^{\prime}=y_{k}^{\prime}=0$. The $\alpha \cdot u_{1}$ term has the property that the symbol for $u_{1}$ is smooth in the variables $x$ and $y_{k}^{\prime}$, so the Taylor series of $a$ as a function of $x / y_{k}^{\prime}$ and $y_{k}^{\prime}$ has the property

$$
\text { The coefficient of }\left(x / y_{k}^{\prime}\right)^{j} y_{k}^{\prime l} \text { vanishes whenever } l<j \text {. }
$$

Thus, the sum of the two terms $u_{0}$ and $\alpha \cdot u_{1}$ has property (5.2). It is clear that this property is not invariant under multiplication by smooth functions of $y_{k}^{\prime}$ and $x / y_{k}^{\prime}$. However, the space $I_{\mathrm{s} \Phi}^{m, m+1 / 2}(Y, L)$ is by its definition invariant under $\mathcal{C}^{\infty}(Y)$. So $I_{\mathrm{sc}}^{m}\left(X,\left(L_{2}, L_{1}\right)\right)$ is a strictly smaller space than $I_{\mathrm{s} \Phi}^{m, m+1 / 2}(Y, L)$. This completes the proof of the Theorem.

Remark. The absence of terms (5.2) in the symbol of $I_{\mathrm{sc}}^{m}\left(X,\left(L_{2}, L_{1}\right)\right)$ is reflected in the symbol calculus for $u \in I_{\mathrm{sc}}^{m}\left(X,\left(L_{2}, L_{1}\right)\right)$. That is, if the symbol $\sigma_{L_{1}}^{m}(u)$ of $u$ on $L_{1}$ vanishes, then $u \in I_{\mathrm{sc}}^{m+1}\left(X,\left(L_{2}, L_{1}\right)\right)+I_{\mathrm{sc}}^{m+1 / 2}\left(X, L_{2}\right)$ (see 
[8], equation (5.2)). On the other hand, if the symbol of $u \in I_{\mathrm{s} \Phi}^{m, m+1 / 2}(Y, L)$ at $L$ vanishes, then $u \in I_{\mathrm{s} \Phi}^{m+1, m+1 / 2}(Y, L)$ (as opposed to $I_{\mathrm{s} \Phi}^{m+1, m+3 / 2}(Y, L)+$ $\left.I_{\mathrm{s} \Phi}^{\infty, m+1 / 2}(Y, L)\right)$. This better vanishing property of intersecting Legendre distributions makes them more useful for analyzing principal type propagation.

\section{Acknowledgment}

The authors are grateful to Rafe Mazzeo and Richard Melrose for helpful discussions, and the referee for several suggestions for improving the quality of the exposition. They acknowledge the support of the Australian Research Council (A. H.) and the National Science Foundation through NSF grant \#DMS99-70607 (A. V.).

\section{References}

[1] V. Guillemin and G. Uhlmann, Oscillatory integrals with singular symbols, Duke Math. J. 48 (1981), 251-267.

[2] A. Hassell, Distorted plane waves for the 3 body Schrödinger operator, Geom. Funct. Anal. 10 (2000), 1-50.

[3] A. Hassell and A. Vasy, The spectral projections and the resolvent for scattering metrics, J. Anal. Math 79 (1999), 241-298.

[4] _ The resolvent for Laplace-type operators on asymptotically conic spaces, Ann. Inst. Fourier, to appear.

[5] L. Hörmander. The analysis of linear partial differential operators, vol. 1-4, SpringerVerlag, 1983.

[6] M. S. Joshi, A symbolic construction of the forward fundamental solution of the wave operator, Comm. Partial Differential Equations 23 (1998), 1349-1417.

[7] R. Mazzeo and R. B. Melrose, Pseudodifferential operators on manifolds with fibred boundaries, Asian J. Math. 2 (1998), 833-866.

[8] R. B. Melrose and G. A. Uhlmann, Lagrangian intersection and the Cauchy problem, Comm. Pure Appl. Math. 32 (1979), 483-519.

[9] R. B. Melrose and M. Zworski, Scattering metrics and geodesic flow at infinity, Invent. Math. 124 (1996), 389-436.

Centre for Mathematics and its Applications, Australian National University, Canberra ACT 0200, Australia.

E-mail address: hassell@maths.anu.edu.au

Department of Mathematics, Massachusetts institute of Technology, Cambridge, MA 02139.

E-mail address: andras@math.mit.edu 